\title{
Computational drug re-purposing studies on SARS-CoV-2 protein targets
}

\author{
Guangfeng Zhou ${ }^{1,2}$, Lance Stewart ${ }^{1,2}$, Gabriella Reggiano ${ }^{1,2}$, and Frank DiMaio ${ }^{1,2, *}$ \\ ${ }^{1}$ Biochemistry Department, University of Washington, Seattle, WA, 98195 \\ ${ }^{2}$ Institute for Protein Design, University of Washington, Seattle, WA, 98195 \\ *Email: dimaio@uw.edu
}

May 15, 2020

\begin{abstract}
Note: The research we report here is purely computational in nature. We have not tested any of the molecules in vitro or in vivo. With the sole exception of Remdesivir, NONE of these molecules have received FDA approval as drugs for treatment of COVID-19 or associated SARS-CoV-2 coronavirus. This is an ongoing work and will be updated frequently.
\end{abstract}

\begin{abstract}
To contribute to the combat of COVID-2019, we applied structure-based computational docking screens using flexible docking protocol of Rosetta GALigandDock against multiple potential SARS-CoV-2 protein targets, including the Nsp5 3-chymotrypsin-like protease (3CLpro), the Nsp3 ADP ribose phosphatase, the Nsp15 Endoribonuclease, the RNA binding domain of nucleocapsid phosphoprotein, the Nsp16 2'-O-MTase, Nsp14, and Nsp12 RNA-dependent RNA polymerase. Screening against a re-purposing library of 8,395 FDA approved drugs at various stages of drug development and various natural products from DrugBank, we found a total of 124 putative inhibitors with predicted binding $\Delta \mathrm{G}$ less than $-8.9 \mathrm{kcal} / \mathrm{mol}$, including HIV-AIDS drugs Nelfinavir and Tipranavir, targeting 3Clpro with $\Delta \mathrm{G}=-18.8 \mathrm{kcal} / \mathrm{mol}$ and $\Delta \mathrm{G}=-16.6$ $\mathrm{kcal} / \mathrm{mol}$ respectively. These primarily involve binders to the Nsp5 3CLpro (37 hits) and the Nsp3 ADP ribose phosphatase (36 hits), with smaller numbers of hits to other targets. These small molecule putative inhibitors suggest a possible avenue for drug repurposing, and the identified compounds should serve as a high-priority list for experimental validation via co-crystallization, enzymatic and cell based assays.
\end{abstract}

\section{Introduction}

Coronaviruses are a large family of viruses that usually cause mild to moderate upper-respiratory tract illnesses. Among the hundreds of known coronaviruses, most only circulate among animals, however, sometimes a spillover event can happen when those viruses jump to human and can cause disease. To this date, there are three known coronaviruses that can cause serious outcomes in people and they are SARS (severe acute respiratory syndrome), which emerged in late 2002; MERS (Middle East respiratory syndrome), which emerged in 2012; and COVID-19, which emerged in December 2019, and found to be associated with a novel coronavirus named SARS-CoV-2[1]. The emergence and rapid increase in cases of COVID-19 has posed great challenges to global public health, research and medical communities. Declared a global pandemic by the World Health Organization on March 11, 2020, COVID-19 has so far infected over 4.7 million people and claimed nearly 300,000 lives. In response to the outbreak, a global effort of research is under way to study and combat this novel coronavirus. At present, Remdesivir is only drug that has received emergency use authorization from the Food and Drug Administration (FDA), limited for use in hospitalized patients with "severe disease" [2]. There are no clinically proven or approved vaccines yet available for COVID-19, though several are under development. 
International leaders have been asked to "solve the immediate problem and keep it from happening again" [3].

One avenue of research for treatment has been exploration of in silico drug discovery and drug re-purposing. Two computational studies based on machine learning models have been published: Zhavoronkov et al. [4] predicted several novel inhibitor molecules using a generative deep learning model, while Nguyen et al.[5] screened 1465 FDA approved drugs from DrugBank[6] using an structural-based drug repositioning (SBDR) model developed by them. In both studies, the target protein was the SARS-CoV-2 3CL protease, where a homology model constructed from SARS 3CL protease X-ray crystal structure (95.25\% sequence identity) was used. Another computational study discovered potential inhibitors for SARS-CoV-2 by docking the ZINC drug database and their in-house natural products database against 21 SARS-CoV-2 targets (19 of which are homology models)[7]. In early February 2020, Jin et al. released the first crystal structure of SARS-CoV-2 3CL protease at $2.16 \AA$ resolution (PDB id: 6LU7) [8]. Shortly after this structure was determined, several groups working at the Diamond light source solved the same structure (PDB id: $6 \mathrm{YB} 7$ ), in addition to performing a large crystallographic fragment screen. They discovered many small molecule fragment hits bound to 3CL protease (PDB ids: 5R7Y, 5R7Z, 5R80, 5R81, 5R82, 5R83, 5R84). Finally, while much of the computational drug design studies have focused on 3CL protease[9], many crystal structures of other protein targets have also been recently determined, including the Nsp3 ADP ribose phosphatase (ADRP) (PDB ids: 6VXS, 6W02, 6WCF[unpublished]), the Nsp15 Endoribonuclease (PDB ids: 6VWW, 6W01[10]), the RNA binding domain of nucleocapsid phosphoprotein (N-RBD) (PDB id: 6VYO[unpublished]) and the Nsp16 2'-O-MTase (PDB id: 6W4H[unpublished]), the Nsp12 RNA-dependent RNA polymerase (RdRp) (PDB id: 6M71[11], 7BV2[12]) and the spike glycoprotein receptor-binding domain bound with the human cellular target ACE-2 (PDB id:6M0J)[13].

Turning novel designed inhibitors into drugs requires a very long period of drug development and clinical trials, so one method for finding potential drugs with a possibility for rapid development is through drug repurposing $[14,15]$. In this manuscript, using a flexible docking protocol in Rosetta, GAligandDock[unpublished], we screen a library of 8,395 compounds from DrugBank[6], which includes both FDA approved drugs at various stages of drug development as well as various natural products, against the above mentioned enzymatic and nonstructural protein targets. This computational screen reveals several dozen compounds predicted as potential inhibitors of SARS-CoV-2, with predicted binding $\Delta \mathrm{G}$ less than $-8.9 \mathrm{kcal} / \mathrm{mol}$, and specific hydrogen-bonding interactions to the binding pocket. Most of these hits are focused on two targets in particular: the SARS-CoV-2 Nsp5 3CLpro (main protease), where we get 15 hits to the 8 available crystal structures; and the Nsp3 ADP ribose phosphatase where we get 9 hits against the apo crystal structure.

\section{Results and Discussion}

Using our newly developed, currently unpublished, Rosetta-based ligand docking forcefield and docking methodology (GALigandDock), we docked the DrugBank library of FDA-approved drugs and natural products against 8 different crystal structures of SARS-CoV-2 protein targets. Briefly, GALigandDock expands Rosetta's per-atom parameters to all of chemical space, and uses a genetic algorithm to simultaneously refine ligand conformations and all pocket sidechains. The best-predicted binders from our computational docking screen are highlighted in Tables 2 to 6. For some targets (e.g., the Nsp5 3CLpro), we use multiple receptor crystal structures to explore target backbone flexibility, and a few hits from our docking screens are hitting multiple receptor structures. Table 2 indicates the PDB id of the target against which the lowest-energy docked configuration was found.

Following docking with GALigandDock, we rank docked poses by predicted binding affinity $(\Delta \mathrm{G})$, and — for the 100 top-ranked structures - calculate the number of buried unsatisfied hydrogen bonds. Manual inspection was then carried out for the top 10 models, and any structures ranked 11-100 with less than 3 unsatisfied hydrogen bonds. Manual inspection eliminated otherwise low-energy binders that 1) did not bind in the pocket of interest (e.g. known active site or substrate binding site) and 2) did not make specific polar or hydrogen-bonding interactions to the target. Our final list contains 124 total hits, all of which have $\Delta \mathrm{G}$ less than $-8.9 \mathrm{kcal} / \mathrm{mol}$, and all but one of which have less than 3 buried unsatisfied hydrogen bonds, as calculated by Rosetta. Focusing on individual targets, we see:

- 37 hits for the Nsp5 3CLpro (9 to 6LU7, 8 to 5R7Y, 1 to 5R7Z, 5 to 5R81, 3 to 5R82, 6 to $5 \mathrm{R} 83,4$ to 
$5 \mathrm{R} 84$ and 1 to $6 \mathrm{~W} 63)$

- 36 hits for the Nsp3 ADP ribose phosphatase (14 to 6VXS, 16 to $6 \mathrm{~W} 02$ and 6 to $6 \mathrm{WCF}$ )

- 17 hits for the Nsp15 Endoribonuclease (9 to 6VWW and 8 to 6W01)

- 3 hits for the RNA binding domain of nucleocapsid phosphoprotein

- 11 hits for the Nsp16 2'-O-MTase

- 7 hits for the Nsp14

- 13 hits for the Nsp12 RNA dependent RNA polymerase (10 to 6M71, 3 to 7BV2)

The disproportionately large number of hits to primarily two targets (Nsp5 3CLpro and Nsp3 ADP ribose phosphatase) is likely due to two reasons: 1) the number of available crystal structures providing alternate backbone conformations for docking and 2) the docking difficulty of the binding site. The Nsp5 3CLpro target has the largest number of hits since there are eight high resolution crystal structures available, and the binding pocket of Nsp5 3CLpro is also well defined and relatively deep making it an easier target for docking (see Figure 1 ). Although there are only three crystal structure for the Nsp3 ADP ribose phosphatase, it also has a large number of hits due to a deep, well defined pocket, where the ADP ribose binds. Considering $\Delta$ Gs, these two targets also tend to have better binding affinities as well, suggesting they might be easier targets for small molecules to bind (and consequently inhibit) generally. In addition, Figure $2 \mathrm{a}-\mathrm{g}$ shows the predicted bound conformation of the best-predicted binders to each of the seven screened targets. Note that for all seven cases, extensive hydrogen bond interactions occur between ligand and receptor (yellow dashed lines in Figure 2). Additionally some targets (e.g., Panels b) feature favorable hydrophobic (ring-stacking interactions) between the protein and ligand.

An analysis of some of the most interesting hits follows. The top ranked hit (DB12116) to Nsp5 3CLpro, Epigallocatechin gallate, which is the major tea catechin, has been reported to exhibit anti-inflammatory, antioxidative, anti-apoptotic, antiviral potentials[16-20]. More interestingly, it has been shown that HIV-1 protease kinetics were suppressed by it at a concentration higher than $10 \mu \mathrm{M}$ in a cell-free study[20]. Another interesting hit, DB00220, or Nelfinavir, an HIV-1 protease inhibitor developed by Agouron Pharmaceuticals (now part of Pfizer) approved by FDA in 1997 [21], was ranked 11th on our list (and 2nd overall in docking $\Delta$ Gs against the protease target structure 5R7Y) (Figure 3(a)) and was independently found by computational approaches to be a candidate inhibitor of 3CLpro[22]. DB00932, or Tipranavir, ranking 24th on our list (and 14 th overall in docking $\Delta$ Gs against the protease target structure 6LU7) also demonstrated a reasonable pose (Figure 3(b)), is an FDA approved (2005) non-peptidic protease inhibitor to treat HIV-1, originally discovered by researchers at Pharmacia \& Upjohn (now Pfizer) in the US, and co-developed with Boehringer Ingelheim[23]. Both Nelfinavir (Vriacept ${ }^{\circledR}$, Pfizer) and Tipranavir (Aptivus ${ }^{\circledR}$, Boehringer Ingelheim) are oral pill therapeutics.

In addition to the Nsp5 3CLpro target, there are some interesting trends in hits to other targets. Figures 5 to 11 shows the $2 \mathrm{D}$ structure depictions of the manually selected small molecules for each target. A majority of structures involve aromatic ring interactions and extensive hydrogen bond networks (some nice examples are shown in Figure 2). Phosphate groups are highly represented: among the hits to the target of Nsp3 ADP ribose phosphatase, four of them (DB04294, DB04733, DB03116, DB02098) have multiple phosphate groups and they are making extensive hydrogen bonds with a cluster of backbone NH groups (VAL49, ILE131, PHE156, ASP157; see Figure 12a). Similarly, the phosphate group in the hits (DB02711, DB03532, DB04516) to Nsp15 Endoribonuclease are coordinated by the same receptor residues (GLY248, HIS250, LYS290, THR341). The hit DB02711 also has stacking interactions with receptor TRP333 (see Figure 12b).

RNA-dependent RNA polymerase (Nsp12 RdRp) and the proofreading exonuclease (Nsp14 ExoN, not included in our analysis) [24] are both antagonized by the metabolites of Remdesivir (GS-5734), a monophosphoramidate prodrug of an adenosine analogue developed by Gilead Sciences as an intravenous treatment for Ebola virus disease [25]. Remdesivir is only drug so far to have received emergency use authorization from the Food and Drug Administration (FDA), limited for use in hospitalized patients with "severe disease" (May 2, 2020). Remdesivir 
has been reported to have an EC90 value against SARS-CoV-2 in Vero E6 cells of 1.76 $\mu \mathrm{M}$ which may be of pharmacological relevance for slowing disease progression or speeding recovery from COVID-19 [26]. Biochemical and modeling studies suggest that the mechanism of action for Remdesivir for RdRp is delayed chain termination following incorporation of the the GS-441524-MP 5' monophosphate nucleoside metabolite of Remdesivir $[27,28]$. Our GALigandDock analysis RdRp included the DrugBank set of compounds which contains Remdesivir GS-5734 (which as a pro-drug molecule was not predicted to bind RdRp), as well as GS-441524-TP the '5 triphosphate active metabolite of Remdesivir and the GS-441524-MP 5' monophosphate nucleoside metabolite. Our GALigandDock was run for the Nsp12 RdRp structure 6M71 and the 7BV2 structure with RNA substrate where we intentionally removed the covalently bound Remdesivir monophsphate that is incorporated into the primer strand at the first replicated base pair. We observed similar low energy binding modes of DB03755 tetraphosphate Adenosine-5'-[Beta, Gamma-Methylene] Tetraphosphate and the GS-441524-TP binding into the pocket of apo $\operatorname{RdRp}(6 \mathrm{M} 71)$ that would otherwise be occupied by primer strand incorporated Remdesivir monophosphate (GS-441524-MP) in 7BV2. For the Remdesivir removed 7BV2 structure we observed a low energy binding mode for DB01082 Streptomycin, see 2(g) and 3(c,d).

Chloroquine, a widely-used anti-malarial and autoimmune disease drug, has received significant attention, as it may be of potential utility in treating COVID-19. In vitro, chloroquine has an EC90 value of $6.90 \mu \mathrm{M}$ for inhibiting viral replication of SARS-CoV-2 in Vero E6 cells [26]. Use of chloroquine to treat COVID-19 patients is very controversial, with only limited and clinical data on its efficacy or lack thereof[29]. While chloroquine was included in our computational modeling, we did not observe any calculated low energy binding modes to any of the SARS-CoV-2 targets we studied. The anti-viral mechanism of action for chloroquine is based on its ability to antagonize endosomal viral / cellular membrane fusion by virtue of its ability to increase endosomal $\mathrm{pH}$, as well as its ability to interfere with the glycosylation of the coronavirus cellular receptor ACE-2[30]. Considering these points, we view chloroquine as an interesting negative control for our GALigandDock studies.

Finally, we compare our docking results to the ligand screens against the SARS-CoV-2 protease against the DSI-poised fragment library. In Figure 4, we superimpose the predicted docking pose of some hits (shown in purple) with the fragments (panel a \& b) and the ligand (panel c to f) in crystal structures (shown in cyan). There is an obvious structural similarity between the predicted docking poses to the crystal structures. Figure 4 (a) \& (b) shows that the predicted pose of two hits to Nsp5 3CLpro closely resemble the fragments in the crystal structures. In (a), our computationally docked model of DB12116 (Epigallocatechin gallate) places two aromatic rings in the same region as two fragments (PDB ids: 5R7Y and 5R83). In (b) the pyridine group in our computationally docked model of DB00884 (Risedronic acid) overlaps perfectly with a fragment pyridine group (PDB id: 5R83), and the model adds phosphate groups making extensive hydrogen bonds with the receptor. Moreover, in Figure 4(c) to (f), the predicted docking poses of has nearly identical binding mode compared to the ligand structures in the crystal structure of Nsp16 2'-O-MTase (PDB id: 6W4H), Nsp14 (PDB id: 5C8T) and Nsp3 ADRP (PDB id: 6W02).

\section{Conclusions}

In this work, we used Rosetta GALigandDock to screen a 8,395-compounds library of FDA approved drugs at various stages of drug development and various natural products from DrugBank against several SARS-CoV-2 protein targets. Docking was carried out with a full sidechain flexibility in the active site, against all potential SARS-CoV-2 protein targets for which structures are currently available. Based on the docking results, we identify 124 promising targets that we recommend for further experimental investigation.

We think this work is most valuable for researchers looking to identify targets to carry through to drug repurposing studies. The computational studies provide a predicted rank ordering of binding affinities which can serve as a resource for prioritizing compounds and targets for further experimental characterization. The calculated binding modes of these small molecule ligands may also inspire drug design targeting inhibition of the defined SARS-CoV-2 targets. As additional X-Ray crystal structures of SARS-CoV-2 targets are solved, or structures are determined with alternate small molecules bound, we will continue to update this manuscript. 


\section{Materials and Methods}

Target structures were downloaded from the Protein Data Bank (PDB). PDB id for Nsp5 3CLpro: 6LU7[8], 6W63, 5R7Y, 5R7Z, 5R81, 5R82, 5R83, 5R84[unpublished]; PDB id for Nsp3 ADP ribose phosphatase from SARS CoV-2: 6VXS, 6W02, 6WCF[unpublished]; PDB id for Nsp15 Endoribonuclease: 6VWW, 6W01 [10]; PDB id for RNA binding domain of nucleocapsid phosphoprotein: 6VYO[unpublished]; PDB id for Nsp16 2'-O-MTase: 6W4H [unpublished]; PDB id for SARS Nsp14: 5C8T; PDB id for Nsp12 RdRp: 6M71[11] ${ }^{1}$, $7 \mathrm{BV} 2[12]$.

\subsection{Ligand preparation.}

SDF files of 8,395 FDA-approved and natural products were acquired from DrugBank[6]. Phenix eLBOW[32] was used to preprocess the ligands, predicting the protonation states at physiological pH. Next, antechamber from AMBER Tools 19.0[33] was used to obtain the AM1-BCC partial charges for each atom. Finally, mol2genparams.py (part of Rosetta's scripts repository) was used to generate the Rosetta parameter files for all the compounds.

\subsection{Molecular docking.}

FDA approved drugs at various stages of drug developments and various natural products (8,395 compounds) were docked into the active site of SARS-CoV-2 Nsp5 3CLpro, Nsp3 ADP ribose phosphatase, Nsp15 Endoribonuclease, RNA binding domain of nucleocapsid phosphoprotein, Nsp16 2'-O-MTase, homology model of Nsp14 constructed using SARS Nsp14 structure (sequence identity $>90 \%$, constructed using RosettaCM [34] ) and Nsp12 RdRp using Rosetta GALigandDock[unpublished]. To prepare the protein crystal structures for docking, all the water molecules, ions and other organic solvent molecules were removed. The active site of Nsp12 RdRp is defined by both protein and RNA, however in crystal structure 6M71, RNA is not present. We added RNA structure from HCV ns5b (4WTG) to the model and refined with Rosetta's relax. Flexible side chains in the active site were automatically determined. The conformation of ligands and flexible side chains were optimized simultaneously using genetic algorithm coupled with Rosetta grid energy function. Protein backbones in the active site along with ligands and flexible side chains were optimized in the final round of docking using Rosetta full energy function. Binding affinity were estimated for each binding pose and the pose with the best binding affinity was saved. After completing the docking screens, top-ranked 100 structures were chosen for further buried unsatisfied hydrogen bond analysis[35] in Rosetta. Rosetta buried unsatisfied hydrogen bond filter reports the difference of the number of buried unsatisfied polar atoms between the bound and unbound state. Any structure except top-ranked ten structures with more than three buried unsatisfied polar atoms was discarded. The rest of the structures (usually around 30 compounds) were manually examined and selected according to the fitness in the pocket, specific polar interactions, etc.

\section{Acknowledgement}

We thank Andrzej Joachimiak and the Center for Structural Genomics of Infectious Disease (https://csgid.org), and Andrew Mesecar, for providing structure coordinates before public release via the Protein Data Bank. We thank Wes Van Voorhis for his input on SARS-CoV-2 targets. We thank Ian Haydon for manuscript assistance. Work was supported by funding from Eric and Wendy Schmidt by recommendation of the Schmidt Futures program, and via The Audacious Project at the Institute for Protein Design.

\section{Supplementary Information}

PDB files of the selected hits are available in supplementary information. (SI_pdbs.zip)

\footnotetext{
${ }^{1}$ RNA in the structure is from HCV ns5b (4WTG) for 6M71 [31].
} 


\section{References}

[1] Peng Zhou et al. "A pneumonia outbreak associated with a new coronavirus of probable bat origin". In: Nature 579.7798 (2020), pp. 270-273.

[2] Jonathan Grein et al. "Compassionate use of remdesivir for patients with severe Covid-19". In: New England Journal of Medicine (2020).

[3] Bill Gates. "Responding to Covid-19 - A Once-in-a-Century Pandemic?" In: New England Journal of Medicine (2020).

[4] Alex Zhavoronkov et al. "Potential COVID-2019 3C-like Protease Inhibitors Designed Using Generative Deep Learning Approaches". In: ChemRxiv (Feb. 2020). DOI: 10.26434/chemrxiv.11829102.v2.

[5] Duc Duy Nguyen et al. "Potentially highly potent drugs for 2019-nCoV". In: bioRxiv (2020).

[6] David S Wishart et al. "DrugBank 5.0: a major update to the DrugBank database for 2018". In: Nucleic Acids Research 46.D1 (Nov. 2017), pp. D1074-D1082.

[7] Canrong Wu et al. "Analysis of therapeutic targets for SARS-CoV-2 and discovery of potential drugs by computational methods". In: Acta Pharmaceutica Sinica B (2020).

[8] Zhenming Jin et al. "Structure of Mpro from COVID-19 virus and discovery of its inhibitors". In: bioRxiv (2020).

[9] Khushboo Bafna, Robert M. Krug, and Gaetano Montelione. "Structural Similarity of SARS-CoV2 Mpro and HCV NS3/4A Proteases Suggests New Approaches for Identifying Existing Drugs Useful as COVID-19 Therapeutics". In: (Apr. 2020). DOI: 10.26434/chemrxiv.12153615.v1.

[10] Youngchang Kim et al. "Crystal structure of Nsp15 endoribonuclease NendoU from SARS-CoV-2". In: Protein Science n/a.n/a (). DOI: 10.1002/pro.3873.

[11] Yan Gao et al. "Structure of the RNA-dependent RNA polymerase from COVID-19 virus". In: Science 368.6492 (2020), pp. 779-782. ISSN: 0036-8075. DOI: 10.1126/science. abb7498.

[12] Wanchao Yin et al. "Structural basis for inhibition of the RNA-dependent RNA polymerase from SARSCoV-2 by remdesivir". In: Science (2020). ISSN: 0036-8075. DOI: 10.1126/science.abc1560.

[13] Jun Lan et al. "Crystal structure of the 2019-nCoV spike receptor-binding domain bound with the ACE2 receptor". In: bioRxiv (2020).

[14] Sudeep Pushpakom et al. "Drug repurposing: progress, challenges and recommendations". In: Nature Reviews Drug Discovery 18.1 (2018), pp. 41-58.

[15] Linda Sleire et al. "Drug repurposing in cancer". In: Pharmacological Research 124 (2017), pp. 74-91.

[16] Massimo Donà et al. "Neutrophil restraint by green tea: inhibition of inflammation, associated angiogenesis, and pulmonary fibrosis". In: The Journal of Immunology 170.8 (2003), pp. 4335-4341.

[17] Emily K Schroeder et al. "Green tea epigallocatechin 3-gallate accumulates in mitochondria and displays a selective antiapoptotic effect against inducers of mitochondrial oxidative stress in neurons". In: Antioxidants \&3 redox signaling 11.3 (2009), pp. 469-480.

[18] Derliz Mereles and Werner Hunstein. "Epigallocatechin-3-gallate (EGCG) for clinical trials: more pitfalls than promises?" In: International journal of molecular sciences 12.9 (2011), pp. 5592-5603.

[19] Mikio Nakayama et al. "Inhibition of the infectivity of influenza virus by tea polyphenols". In: Antiviral Research 21.4 (1993), pp. 289-299.

[20] Koushi Yamaguchi et al. "Inhibitory effects of ()-epigallocatechin gallate on the life cycle of human immunodeficiency virus type 1 (HIV-1)". In: Antiviral Research 53.1 (2002), pp. 19-34.

[21] JS James. "Nelfinavir (Viracept) approved: fourth protease inhibitor available." In: AIDS treatment news 267 (1997), p. 1.

[22] Zhijian Xu et al. "Nelfinavir was predicted to be a potential inhibitor of 2019-nCov main protease by an integrative approach combining homology modelling, molecular docking and binding free energy calculation". In: bioRxiv (2020). 
[23] "Tipranavir". In: Drugs in R $\&$ D 7.1 (2006), pp. 55-62.

[24] Maria L Agostini et al. "Coronavirus susceptibility to the antiviral remdesivir (GS-5734) is mediated by the viral polymerase and the proofreading exoribonuclease". In: MBio 9.2 (2018), e00221-18.

[25] Travis K Warren et al. "Therapeutic efficacy of the small molecule GS-5734 against Ebola virus in rhesus monkeys". In: Nature 531.7594 (2016), pp. 381-385.

[26] Manli Wang et al. "Remdesivir and chloroquine effectively inhibit the recently emerged novel coronavirus (2019-nCoV) in vitro". In: Cell Research 30.3 (2020), pp. 269-271. ISSN: 1001-0602. DOI: 10.1038/s41422020-0282-0.

[27] Calvin J Gordon et al. "The antiviral compound remdesivir potently inhibits RNA-dependent RNA polymerase from Middle East respiratory syndrome coronavirus". In: Journal of Biological Chemistry 295.15 (2020), pp. 4773-4779.

[28] Ashleigh Shannon et al. "Remdesivir and SARS-CoV-2: Structural requirements at both nsp12 RdRp and nsp14 Exonuclease active-sites". In: Antiviral Research (2020), p. 104793.

[29] Jihad Mallat et al. "Hydroxychloroquine is associated with slower viral clearance in clinical COVID-19 patients with mild to moderate disease: A retrospective study". In: medRxiv (2020). DOI: 10.1101/2020. 04.27 .20082180 .

[30] Martin J Vincent et al. "Chloroquine is a potent inhibitor of SARS coronavirus infection and spread". In: Virology journal 2.1 (2005), p. 69.

[31] Todd C. Appleby et al. "Structural basis for RNA replication by the hepatitis C virus polymerase". In: Science 347.6223 (2015), pp. 771-775. ISSN: 0036-8075. DOI: 10.1126/science.1259210. eprint: https: //science.sciencemag.org/content/347/6223/771.full.pdf. URL: https://science.sciencemag. org/content/347/6223/771.

[32] Dorothee Liebschner et al. "Macromolecular structure determination using X-rays, neutrons and electrons: recent developments in Phenix". In: Acta Crystallographica Section D 75.10 (Oct. 2019), pp. 861-877.

[33] D.A. Case et al. AMBER 2019, University of California, San Francisco. 2019.

[34] Yifan Song et al. "High-Resolution Comparative Modeling with RosettaCM". In: Structure 21.10 (2013), pp. 1735-1742.

[35] P Benjamin Stranges and Brian Kuhlman. "A comparison of successful and failed protein interface designs highlights the challenges of designing buried hydrogen bonds." In: Protein science : a publication of the Protein Society 22.1 (2012), pp. 74-82. 
Table 1: Pocket definition by target

\begin{tabular}{ll}
\hline target & pocket residues \\
\hline Nsp5 3CLpro & T25, H41, S46, N142, E166, Q189 \\
Nsp15 Endoribonuclease & H235, T341, S284, K290 \\
Nsp3 ADRP & N40, G48, L126, I131, F156 \\
N-RBD & A55A, T57A, N154B, N75B, D82B \\
Nsp16 2'-O-MTase & D6912, D6897, G6879, N6841, Y6930 \\
Nsp14 & S369, A353, G333, R310, W385, F426, R269 \\
Nsp12 RdRp & D760, P620, D623, S682, U10, U20 \\
\hline
\end{tabular}


Table 2: Docking results for SARS-CoV-2 Nsp5 3CLpro

\begin{tabular}{|c|c|c|c|c|c|c|}
\hline & Target & DrugBank ID & Generic Name & $\mathrm{dG}$ & Docking Rank & Unsat HB \\
\hline 1 & $5 \mathrm{R} 7 \mathrm{Y}$ & DB12116 & Epigallocatechin gallate & -20.241 & 1 & 2 \\
\hline 2 & 6LU7 & DB06255 & Incadronic acid & -20.138 & 1 & 1 \\
\hline 3 & $5 \mathrm{R} 83$ & DB04241 & 1 & -19.806 & 1 & 0 \\
\hline 4 & $5 \mathrm{R} 81$ & DB03495 & 2 & -19.722 & 3 & 2 \\
\hline 5 & $5 \mathrm{R} 83$ & DB03327 & 3 & -19.718 & 2 & 0 \\
\hline 6 & $5 \mathrm{R} 81$ & DB12005 & Nirogacestat & -19.247 & 5 & 2 \\
\hline 7 & $5 \mathrm{R} 83$ & DB04205 & Thymidine-3',5'-Diphosphate & -19.140 & 3 & 2 \\
\hline 8 & $6 \mathrm{~W} 63$ & DB12140 & Dilmapimod & -18.950 & 5 & 5 \\
\hline 9 & 6LU7 & DB03956 & Inositol 2,4,5-trisphosphate & -18.915 & 2 & 1 \\
\hline 10 & $5 \mathrm{R} 82$ & DB08185 & 4 & -18.806 & 2 & 0 \\
\hline 11 & $5 \mathrm{R} 7 \mathrm{Y}$ & DB00220 & Nelfinavir & -18.776 & 2 & 3 \\
\hline 12 & $5 \mathrm{R} 82$ & DB02046 & 5 & -18.560 & 3 & 4 \\
\hline 13 & $5 \mathrm{R} 7 \mathrm{Y}$ & DB04852 & Implitapide & -18.232 & 3 & 0 \\
\hline 14 & $5 \mathrm{R} 83$ & DB03401 & 6 & -18.002 & 8 & 2 \\
\hline 15 & $5 \mathrm{R} 83$ & DB03200 & 7 & -17.975 & 9 & 2 \\
\hline 16 & $5 \mathrm{R} 84$ & DB02819 & 8 & -17.837 & 3 & 1 \\
\hline 17 & $5 \mathrm{R} 81$ & DB11620 & Neridronic Acid & -17.039 & 18 & 1 \\
\hline 18 & $5 \mathrm{R} 84$ & DB01946 & 9 & -17.035 & 15 & 2 \\
\hline 19 & 6LU7 & DB00884 & Risedronic acid & -17.011 & 8 & 0 \\
\hline 20 & $5 \mathrm{R} 7 \mathrm{Z}$ & DB01729 & 10 & -16.829 & 9 & 5 \\
\hline 21 & 6LU7 & DB02552 & Geranyl Diphosphate & -16.825 & 11 & 0 \\
\hline 22 & $5 \mathrm{R} 84$ & DB03576 & 11 & -16.752 & 19 & 1 \\
\hline 23 & 6LU7 & DB04373 & 12 & -16.675 & 14 & 2 \\
\hline 24 & 6LU7 & DB00932 & Tipranavir & -16.602 & 14 & 0 \\
\hline 25 & 6LU7 & DB02808 & Trifluorofurnesyl Diphosphate & -16.266 & 17 & 1 \\
\hline 26 & $5 \mathrm{R} 83$ & DB03433 & 13 & -16.120 & 28 & 1 \\
\hline 27 & 6LU7 & DB02981 & 14 & -16.032 & 21 & 1 \\
\hline 28 & $5 \mathrm{R} 81$ & DB11939 & Henagliflozin & -15.935 & 35 & 2 \\
\hline 29 & $5 \mathrm{R} 81$ & DB12052 & Icariin & -15.923 & 12 & 0 \\
\hline 30 & $5 \mathrm{R} 7 \mathrm{Y}$ & DB02480 & 15 & -15.912 & 13 & 0 \\
\hline 31 & $5 \mathrm{R} 7 \mathrm{Y}$ & DB07300 & 16 & -15.799 & 15 & 2 \\
\hline 32 & $5 \mathrm{R} 82$ & DB04176 & 17 & -15.625 & 19 & 1 \\
\hline 33 & $5 \mathrm{R} 84$ & DB04467 & $\mathrm{N}-\left(5^{\prime}\right.$-phosphopyridoxyl)-L-alanine & -15.188 & 58 & 1 \\
\hline 34 & $5 \mathrm{R} 7 \mathrm{Y}$ & DB02835 & Alpha-D-Glucose 1,6-Bisphosphate & -14.691 & 39 & 1 \\
\hline 35 & $5 \mathrm{R} 7 \mathrm{Y}$ & DB02030 & Alpha-Ribazole-5'-Phosphate & -14.623 & 43 & 0 \\
\hline 36 & 6LU7 & DB12179 & Secoisolariciresinol & -14.061 & 57 & 1 \\
\hline 37 & $5 \mathrm{R} 7 \mathrm{Y}$ & DB06429 & Talnetant & -13.459 & 55 & 0 \\
\hline
\end{tabular}

1 N-Pyridoxyl-2-Methylalanine-5-Phosphate

2 4,6-Dideoxy-4-\{[4,5,6-Trihydroxy-3-(Hydroxymethyl)Cyclohex-2-En-1-Yl]Amino $\}$-Alpha-D-Lyxo-Hexopyranosyl-(1-¿4)-Alpha-DThreo-Hexopyranosyl-(1-¿6)-Alpha-L-Threo-Hexopyranose

3 1-[(3-Hydroxy-Methyl-5-Phosphonooxy-Methyl-Pyridin-4-Ylmethyl)-Amino]-Ethyl $\}$-Phosphonic Acid

4 2-METHYLTHIO-N6-ISOPENTENYL-ADENOSINE-5'-MONOPHOSPHATE

5 N-[(Furan-2-Yl)Carbonyl]-(S)-Leucyl-(R)-[1-Amino-2(1h-Indol-3-Yl)Ethyl]-Phosphonic Acid

6 1D-myo-inositol 1,4,5-trisphosphate

7 7-Alpha-D-Ribofuranosyl-Purine-5'-Phosphate

8 Mono-[3,4-Dihydroxy-5-(5-Methyl-Benzoimidazol-1-Yl)-Tetrahydor-Furan-2-Ylmethyl] Ester

${ }^{9}$ 3-[1-(3-Aminopropyl)-1h-Indol-3-Yl]-4-(1-Methyl-1h-Indol-3-Yl)-1h-Pyrrole-2,5-Dione

${ }_{10}$ (1s,3s, 4s)-1,3,4-Triphospho-Myo-Inositol

11 N-Pyridoxyl-Threonine-5-Monophosphate

12 4-Amino-N-\{4-[2-(2,6-Dimethyl-Phenoxy)-Acetylamino]-3-Hydroxy-1-Isobutyl-5-Phenyl-Pentyl $\}$-Benzamide

13 \{3-[(3-Hydroxy-2-Methyl-5-Phosphonooxymethyl-Pyridin-4-Ylmethyl)-Amino]-2-Methyl-Propyl\}-Phosphonic Acid

14 Vitamin B6 Complexed with 2-Amino-Hexanoic Acid

15 (S)-4-bromo-3-hydroxy-3-methylbutyl diphosphate

16 2-(1H-imidazol-1-yl)-9-methoxy-8-(2-methoxyethoxy) benzo[c] [2,7]naphthyridin-4-amine

17 Phosporic Acid Mono-[3,4-Dihydroxy-5-(5-Methoxy-Benzoimidazol-1-Yl)-Tetrahydro-Furan-2-Ylmethyl] Ester 
Table 3: Docking results for SARS-CoV-2 Nsp15 Endoribonuclease

\begin{tabular}{llllccc}
\hline & Target & DrugBank ID & Generic Name & dG & Docking Rank & Unsat HB \\
\hline 1 & $6 \mathrm{VWW}$ & DB04060 & 1 & -15.417 & 1 & 0 \\
2 & $6 \mathrm{VWW}$ & DB02711 & 2 & -14.314 & 1 & 0 \\
3 & $6 \mathrm{VWW}$ & DB02276 & 3 & -13.705 & 8 & 0 \\
4 & $6 \mathrm{VWW}$ & DB03327 & 4 & -13.504 & 9 & 0 \\
5 & $6 \mathrm{WWW}$ & DB02030 & Alpha-Ribazole-5'-Phosphate & -13.061 & 11 & 0 \\
6 & $6 \mathrm{VWW}$ & DB03532 & 5 & -13.038 & 3 & 0 \\
7 & $6 \mathrm{VWW}$ & DB02992 & 6 & -13.027 & 12 & 0 \\
8 & $6 \mathrm{~W} 01$ & DB02053 & Ribose-5-phosphate & -12.962 & 3 & 1 \\
9 & $6 \mathrm{WWW}$ & DB07652 & 7 & -12.856 & 14 & 0 \\
10 & $6 \mathrm{~W} 01$ & DB03042 & 5 -Phosphoarabinonic Acid & -12.851 & 4 & 1 \\
11 & $6 \mathrm{VWW}$ & DB03401 & 8 & -12.646 & 16 & 0 \\
12 & $6 \mathrm{~W} 01$ & DB01923 & L-Xylulose 5-Phosphate & -12.252 & 6 & 3 \\
13 & $6 \mathrm{~W} 01$ & DB02209 & Pyridoxine phosphate & -11.811 & 7 & 3 \\
14 & $6 \mathrm{~W} 01$ & DB03846 & 9 & -11.070 & 15 & 1 \\
15 & $6 \mathrm{~W} 01$ & DB03464 & Formycin-5'-Monophosphate & -10.715 & 18 & 1 \\
16 & $6 \mathrm{~W} 01$ & DB02630 & L-xylitol 5-phosphate & -10.712 & 19 & 2 \\
17 & $6 \mathrm{~W} 01$ & DB04516 & 10 & -10.633 & 1 & 2 \\
\hline
\end{tabular}

${ }^{1}$ (5-Methyl-6-Oxo-1,6-Dihydro-Pyridin-3-Yl)-1,2-Dideoxy-Ribofuranose-5-Monophosphate

2 4- 2 ,6,8-Trioxo-9-[(2S,3R,4R)-2,3,4,5-Tetrahydroxypentyl]-1,2,3,6,8,9-Hexahydro-7h-Purin-7-Yl $\}$ Butyl Dihydrogen Phosphate

3 (S)-2-(Phosphonoxy)Caproyl-L-Leucyl-P-Nitroanilide

4 \{1-[(3-Hydroxy-Methyl-5-Phosphonooxy-Methyl-Pyridin-4-Ylmethyl)-Amino]-Ethyl\}-Phosphonic Acid

5 Phosphomethylphosphonic Acid Guanylate Ester

6 1-Deoxy-6-O-Phosphono-1-[(Phosphonomethyl)Amino]-L-Threo-Hexitol

7 1-[2-DEOXYRIBOFURANOSYL]-2,4-DIFLUORO-5-METHYL-BENZENE-5'MONOPHOSPHATE

8 D-myo-inositol 1,4,5-trisphosphate

9 5-Hydroxymethyluridine-2'-Deoxy-5'-Monophosphate

10 2-Deoxy-Glucitol-6-Phosphate 
Table 4: Docking results for SARS-CoV-2 Nsp3 ADP ribose phosphatase

\begin{tabular}{|c|c|c|c|c|c|c|}
\hline & Target & DrugBank ID & Generic Name & $\mathrm{dG}$ & Docking Rank & Unsat HB \\
\hline 1 & 6W02 & DB02738 & Adenosine-5'-Pentaphosphate & -38.405 & 1 & 11 \\
\hline 2 & 6W02 & DB02319 & 5,6-Dihydroxy-Nadp & -36.957 & 2 & 9 \\
\hline 3 & 6W02 & DB02059 & Adenosine-5-Diphosphoribose & -33.660 & 4 & 9 \\
\hline 4 & 6W02 & DB03797 & 1 & -33.489 & 5 & 6 \\
\hline 5 & 6W02 & DB02930 & 2 & -32.591 & 6 & 8 \\
\hline 6 & 6W02 & DB03075 & (Diphosphono)Aminophosphonic Acid & -31.986 & 7 & 2 \\
\hline 7 & 6W02 & DB01975 & CPR & -31.620 & 8 & 6 \\
\hline 8 & 6W02 & DB03893 & 3 & -31.361 & 9 & 7 \\
\hline 9 & 6W02 & DB06255 & Incadronic acid & -30.806 & 10 & 4 \\
\hline 10 & 6W02 & DB03161 & 4 & -30.576 & 11 & 6 \\
\hline 11 & $6 \mathrm{~W} 02$ & DB04983 & Denufosol & -30.357 & 13 & 6 \\
\hline 12 & 6W02 & DB03069 & 5 & -29.678 & 14 & 6 \\
\hline 13 & 6W02 & DB02363 & 6 & -29.651 & 15 & 3 \\
\hline 14 & 6W02 & DB04097 & 7 & -29.198 & 17 & 6 \\
\hline 15 & 6W02 & DB01895 & 8 & -28.914 & 19 & 4 \\
\hline 16 & 6W02 & DB03687 & 9 & -28.810 & 20 & 9 \\
\hline 17 & $6 \mathrm{VXS}$ & DB13764 & Monoxerutin & -22.691 & 4 & 1 \\
\hline 18 & $6 \mathrm{VXS}$ & DB04703 & Hesperidin & -21.908 & 5 & 1 \\
\hline 19 & $6 \mathrm{VXS}$ & DB14128 & Nadide & -21.532 & 7 & 4 \\
\hline 20 & $6 \mathrm{VXS}$ & DB01763 & 10 & -21.376 & 8 & 5 \\
\hline 21 & $6 \mathrm{VXS}$ & DB04294 & 11 & -20.693 & 11 & 2 \\
\hline 22 & $6 \mathrm{VXS}$ & DB02338 & 12 & -20.282 & 15 & 5 \\
\hline 23 & $6 \mathrm{VXS}$ & DB03186 & U-Pi-a-Pi & -20.105 & 18 & 7 \\
\hline 24 & $6 \mathrm{VXS}$ & DB13014 & Hypericin & -20.076 & 8 & 1 \\
\hline 25 & $6 \mathrm{WCF}$ & DB07873 & Lauryl alcohol diphosphonic acid & -19.059 & 5 & 4 \\
\hline 26 & $6 \mathrm{VXS}$ & DB04733 & 13 & -17.701 & 24 & 1 \\
\hline 27 & $6 \mathrm{VXS}$ & DB01024 & Mycophenolic acid & -17.045 & 38 & 1 \\
\hline 28 & $6 \mathrm{VXS}$ & DB01732 & 14 & -17.003 & 40 & 2 \\
\hline 29 & $6 \mathrm{WCF}$ & DB03433 & 15 & -16.692 & 23 & 2 \\
\hline 30 & $6 \mathrm{WCF}$ & DB07894 & 16 & -16.531 & 25 & 1 \\
\hline 31 & $6 \mathrm{VXS}$ & DB03116 & 17 & -16.257 & 65 & 0 \\
\hline 32 & $6 \mathrm{WCF}$ & DB07652 & 18 & -16.112 & 44 & 3 \\
\hline 33 & $6 \mathrm{WCF}$ & DB03486 & 19 & -16.094 & 45 & 3 \\
\hline 34 & $6 \mathrm{VXS}$ & DB06314 & SGX-523 & -16.067 & 68 & 1 \\
\hline 35 & $6 \mathrm{WCF}$ & DB01995 & 5-Methylcytidine-5'-Monophosphate & -15.771 & 63 & 2 \\
\hline 36 & $6 \mathrm{VXS}$ & DB02098 & Adenosine-2'-5'-Diphosphate & -15.234 & 109 & 0 \\
\hline $\begin{array}{ll}1 & 3 \text {-An } \\
2 & \text { Ader } \\
3 & \text { Thio } \\
4 & \text { Thyr } \\
5 & \text { Cyti } \\
6 & 2 \text {-M } \\
7 & \text { Urid } \\
8 & \text { Aspa } \\
9 & 4 \text {-Di } \\
10 & 7 \text {-th } \\
11 & 5 \text {-P } \\
12 & \text { Nad } \\
13 & 1,6- \\
14 & \text { (4r, } \\
15 & \{3-- \\
16 & 4-(2 \\
17 & 5-(1 \\
18 & 1-[2 \\
19 & \text { Pho }\end{array}$ & $\begin{array}{l}\text { inomethyl- } \\
\text { osine 5'-[ } \gamma- \\
\text { hicotinamid } \\
\text { aidine-5'-D } \\
\text { line-5'-Dipl } \\
\text { nnophospho } \\
\text { ne-5'-Diph } \\
\text { rtyl-Adeno } \\
\text { hosphocyt } \\
\text { ionicotinan } \\
\text { osphoribos } \\
\text { ph Dihydrc } \\
\text { DI-O-PHOs } \\
\text { ss,6s,7r)-1,3 } \\
\text { (3-Hydroxy } \\
\text {-HYDROX } \\
\text {-Carboxy-1 } \\
\text {-DEOXYRI } \\
\text { sphomethy] }\end{array}$ & $\begin{array}{l}\text { Pyridinium-Adenin } \\
\text { hio]triphosphate } \\
\text { e-Adenine-Dinucleo } \\
\text { phospho-Beta-D-Xy } \\
\text { ospho-Beta-D-Xylo } \\
\text { adenosine-5'-Dipho } \\
\text { sphate-4-Deoxy-4-H } \\
\text { ine-5'-Monophosph } \\
\text { dyl-2-C-Methyl-D- } \\
\text { ide-adenine-dinucle } \\
\text { l-1-(Beta-Methyle } \\
\text {-Nicotinamide-Ade } \\
\text { PHONO-D-MANN } \\
\text {-Dibenzyl-4,7-Bis(F } \\
\text { 2-Methyl-5-Phosph } \\
\text {-4-FLUOROPHEN } \\
\text { Phosphonooxy-Eth } \\
\text { 3OFURANOSYL]- } \\
\text { ohosphonic Acid G }\end{array}$ & $\begin{array}{l}\text { de } \\
\text { sse } \\
\text { hate } \\
\text { uoro-Alpha-D-Galactose } \\
\text { e } \\
\text { ythritol } \\
\text { tide phosphate } \\
\text { ) Pyrophosphate } \\
\text { ne-Dinucleotidephosphate } \\
\text { COL } \\
\text { enoxymethyl)-5,6-Dihydroxy-1,3 Diazepan-2-One } \\
\text { nooxymethyl-Pyridin-4-Ylmethyl)-Amino]-2-Methy } \\
\text { LTHIO)-BUTYLPHOSPHONIC ACID } \\
\text { xyl)-Shikimate-3-Phosphate } \\
\text { 4-DIFLUORO-5-METHYL-BENZENE-5'MONOP } \\
\text { nosyl Ester }\end{array}$ & $\begin{array}{l}\text { Propyl\}-P } \\
\text { OSPHATE }\end{array}$ & sphonic Acid & \\
\hline
\end{tabular}


Table 5: Docking results for SARS-CoV-2 RNA binding domain of nucleocapsid phosphoprotein

\begin{tabular}{cllllcc}
\hline & Target & DrugBank ID & Generic Name & dG & Docking Rank & Unsat HB \\
\hline 1 & 6VYO & DB08399 & Piceatannol & -11.010 & 5 & 1 \\
2 & 6 VYO & DB07652 & 1 & -9.939 & 9 & 2 \\
3 & 6 VYO & DB04195 & Heptulose-2-Phosphate & -8.911 & 22 & 1 \\
\hline
\end{tabular}

Table 6: Docking results for SARS-CoV-2 Nsp16 2'-O-MTase

\begin{tabular}{lllllcc}
\hline & Target & DrugBank ID & Generic Name & dG & Docking Rank & Unsat HB \\
\hline 1 & $6 \mathrm{~W} 4 \mathrm{H}$ & DB02635 & 1 & -15.820 & 7 & 2 \\
2 & $6 \mathrm{~W} 4 \mathrm{H}$ & DB15013 & TAK-243 & -15.594 & 2 & 0 \\
3 & $6 \mathrm{~W} 4 \mathrm{H}$ & DB12295 & Evodenoson & -15.103 & 14 & 1 \\
4 & $6 \mathrm{~W} 4 \mathrm{H}$ & DB02686 & 2 & -14.970 & 15 & 1 \\
5 & $6 \mathrm{~W} 4 \mathrm{H}$ & DB03325 & Tyrosyladenylate & -13.984 & 24 & 2 \\
6 & $6 \mathrm{~W} 4 \mathrm{H}$ & DB04201 & 3 & -13.474 & 12 & 3 \\
7 & $6 \mathrm{~W} 4 \mathrm{H}$ & DB14663 & Ribavirin monophosphate & -13.465 & 35 & 1 \\
8 & $6 \mathrm{~W} 4 \mathrm{H}$ & DB02738 & Adenosine-5'-Pentaphosphate & -13.291 & 38 & 1 \\
9 & $6 \mathrm{~W} 4 \mathrm{H}$ & DB02030 & Alpha-Ribazole-5'-Phosphate & -13.120 & 14 & 2 \\
10 & $6 \mathrm{~W} 4 \mathrm{H}$ & DB00640 & Adenosine & -12.718 & 20 & 0 \\
11 & $6 \mathrm{~W} 4 \mathrm{H}$ & DB03022 & 4 & -12.589 & 54 & \\
\hline
\end{tabular}

${ }^{1} \mathrm{~N}$-[O-Phosphono-Pyridoxyl]-Isoleucine

2 Undecyl-Beta-D-Maltopyranoside

${ }^{3}$ Histidyl-Adenosine Monophosphate

${ }^{4}$ 3-2,6,8-trioxo-9-[(2R,3S,4R)-2,3,4,5-tetrahydroxypentyl]-1,2,3,6,8,9-hexahydro-7H-purin-7- $\gamma$ lpropyl dihydrogen phosphate

Table 7: Docking results for SARS-CoV-2 Nsp14 homology model

\begin{tabular}{lllllcc}
\hline & Target & DrugBank ID & Generic Name & dG & Docking Rank & Unsat HB \\
\hline 1 & 5C8T homology & DB12052 & Icariin & -30.148 & 1 & 3 \\
2 & 5C8T homology & DB01661 & 1 & -25.150 & 3 & 5 \\
3 & 5C8T homology & DB14982 & Miransertib & -22.936 & 8 & 2 \\
4 & 5C8T homology & DB04882 & Edotecarin & -22.757 & 9 & 2 \\
5 & 5C8T homology & DB07413 & 2 & -22.431 & 16 & 7 \\
6 & 5C8T homology & DB03296 & 3 & -21.562 & 19 & 4 \\
7 & 5C8T homology & DB12895 & TD-139 & -20.642 & 26 & 0 \\
\hline
\end{tabular}

1-(5-phospho-D-ribosyl)-ATP

5'-S-[2-(decylamino)ethyl]-5'-thioadenosine

3 '-beta-Sialyl-beta-lactose 
Table 8: Docking results for SARS-CoV-2 Nsp12 RdRp

\begin{tabular}{lllllcc}
\hline & Target & DrugBank ID & Generic Name & dG & Docking Rank & Unsat HB \\
\hline 1 & 6M71 & DB03755 & 1 & -20.256 & 1 & NA \\
2 & 7BV2 & DB01082 & Streptomycin & -15.890 & 5 & NA \\
3 & 6M71 & DB02755 & 2 & -15.528 & 2 & NA \\
4 & 6M71 & DB03296 & 3 & -14.972 & 3 & NA \\
5 & 6M71 & DB11520 & Hygromycin B & -14.959 & 4 & NA \\
6 & 7BV2 & DB02017 & Imidazole-Derived Cellobiose & -14.223 & 9 & NA \\
7 & 6M71 & DB01661 & 4 & -14.202 & 6 & NA \\
8 & 6M71 & DB03942 & Carboxylic PRPP & -11.267 & 9 & NA \\
9 & 7BV2 & NA & GS-441524-TP & -10.988 & 32 & NA \\
10 & 6M71 & DB02738 & Adenosine-5'-Pentaphosphate & -10.263 & 13 & NA \\
11 & $6 \mathrm{M} 71$ & DB01729 & 5 & -10.067 & 17 & NA \\
12 & 6M71 & DB04205 & Thymidine-3',5'-Diphosphate & -9.867 & 19 & NA \\
13 & 6M71 & DB04248 & beta-(1->4)-galactotriose & -9.658 & 20 & NA \\
\hline
\end{tabular}

${ }^{1}$ Adenosine-5'-[Beta, Gamma-Methylene]Tetraphosphate

2 1-3 Sugar Ring of Pentamannosyl 6-Phosphate

3 3'-beta-Sialyl-beta-lactose

${ }^{4}$ 1-(5-phospho-D-ribosyl)-ATP

5 (1s,3s,4s)-1,3,4-Triphospho-Myo-Inositol 


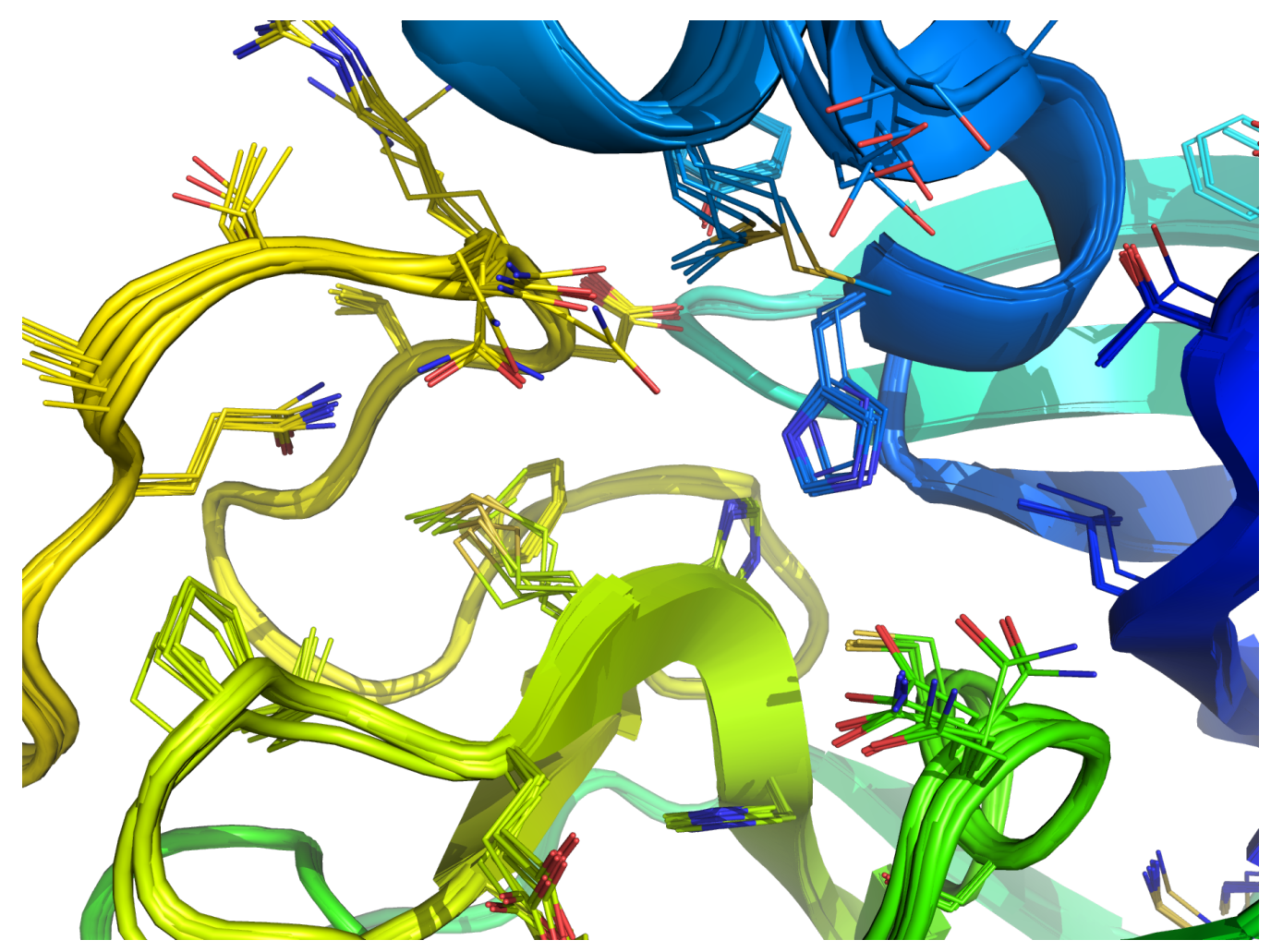

Figure 1: Binding site of superimposed crystal structures of SARS-CoV-2 Nsp5 3CLpro. 


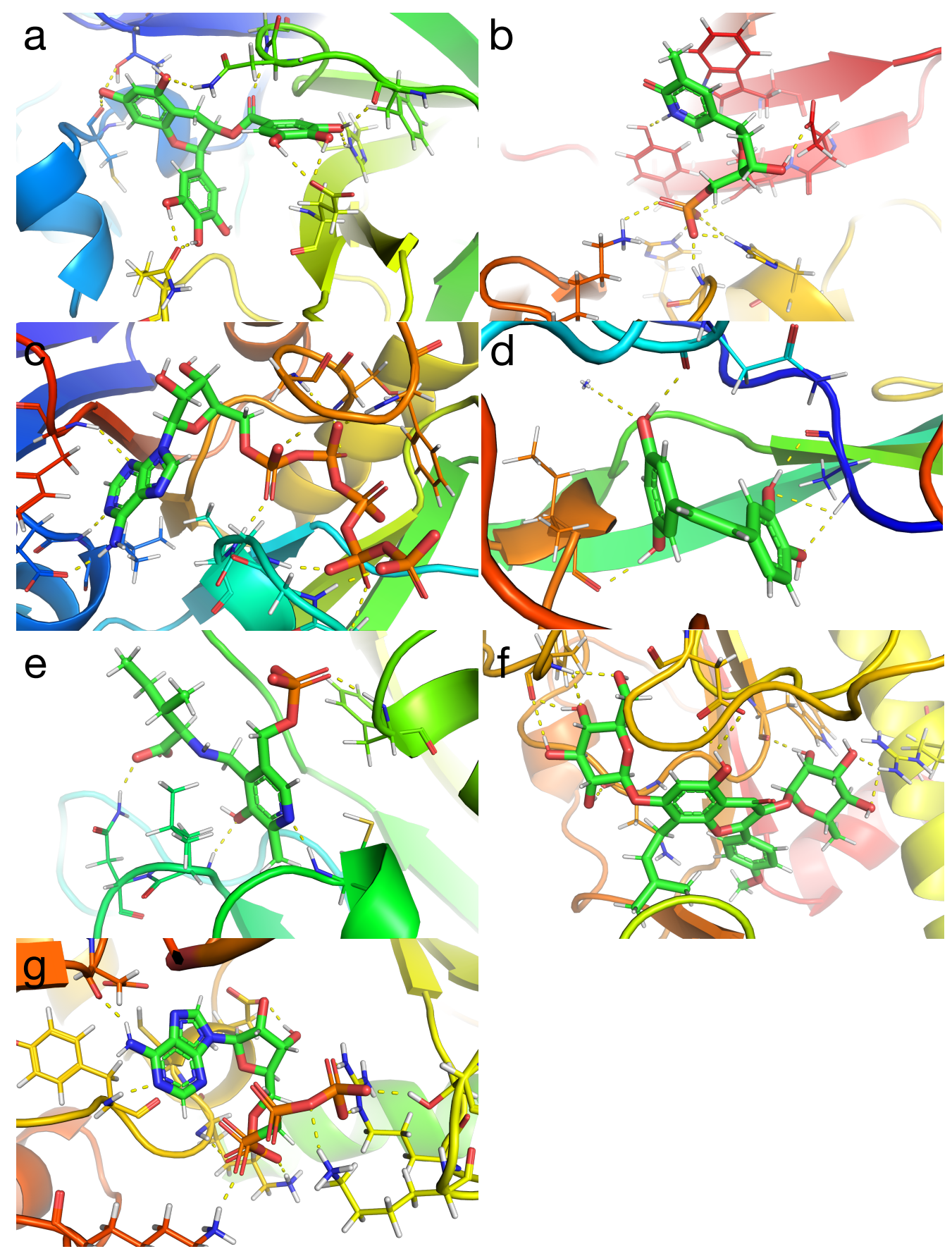

Figure 2: Binding poses of top hit for each target. Yellow dotted lines indicate polar interactions. (a) DB12116 (Epigallocatechin gallate) bound to Nsp5 3CLpro (5R7Y); (b) DB04060 a bound to Nsp15 Endoribonuclease (6VWW); (c) DB02738 (Adenosine-5'-Pentaphosphate) bound to Nsp3 ADP ribose phosphatase (6w02); (d) DB08399 (Piceatannol) bound to Nucleocapsid phosphoprotein (6VYO); (e) DB02635 bound to Nsp16 2'-OMTase (6W4H); (f) DB12052 (Icariin) bound to Nsp14 homology model (5C8T); (g) DB03755c bound to Nsp12 $\operatorname{RdRp}(6 \mathrm{M} 71)$.

a (5-Methyl-6-Oxo-1,6-Dihydro-Pyridin-3-Yl)-1,2-Dideoxy-Ribofuranose-5-Monophosphate

b N-[O-Phosphono-Pyridoxyl]-Isoleucine

c Adenosine-5'-[Beta, Gamma-Methylene]Tetraphosphate 


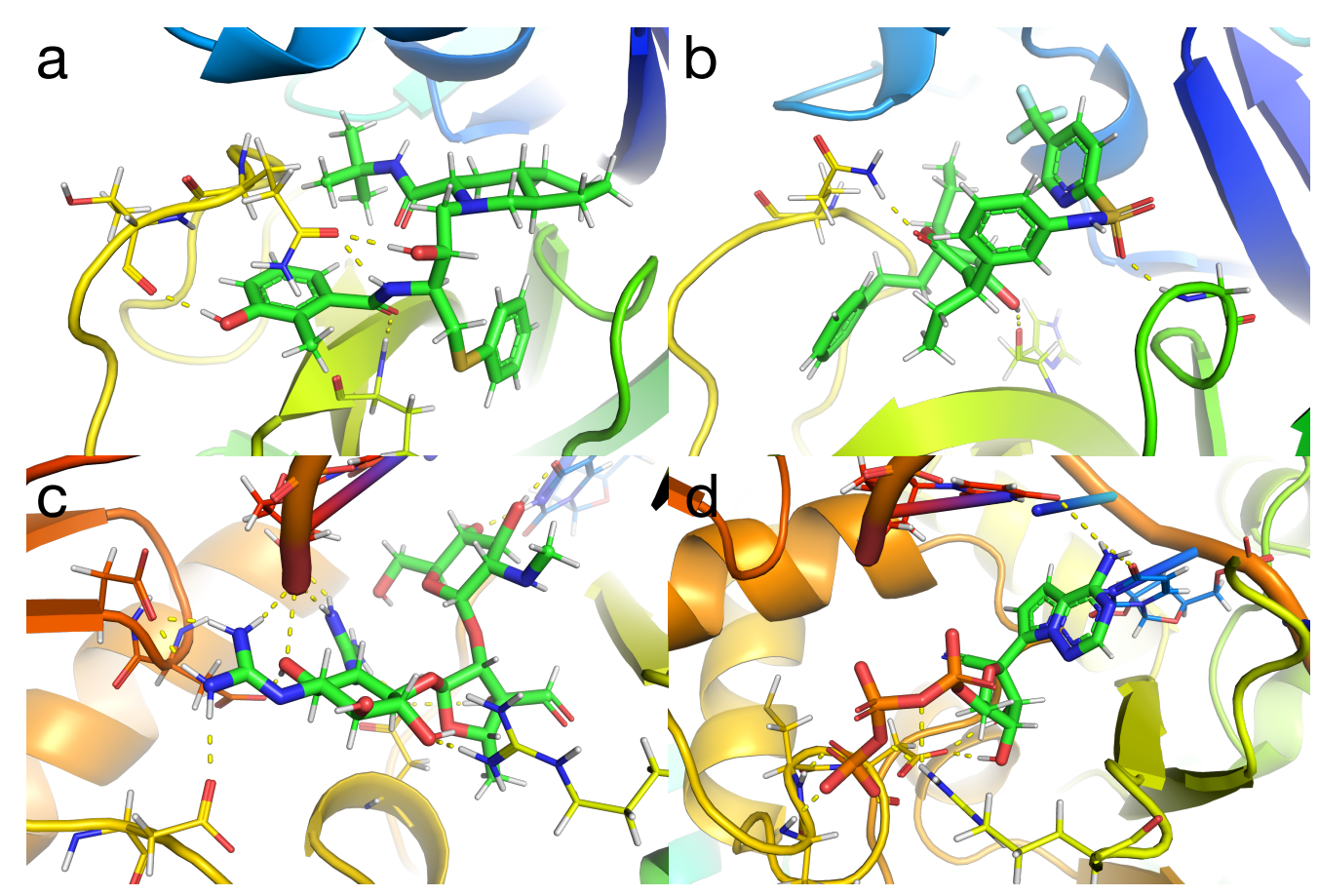

Figure 3: Binding poses of some interesting hits. Yellow dotted lines indicate polar interactions. (a) DB00220 (Nelfinavir) bound to Nsp5 3CLpro (5R7Y); (b) DB00932 (Tipranavir) bound to Nsp5 3CLpro (6LU7); (c) DB01082 (Streptomycin) bound to Nsp12 RdRp (7BV2); (d) GS-441524-TP bound to Nsp12 RdRp (7BV2). 

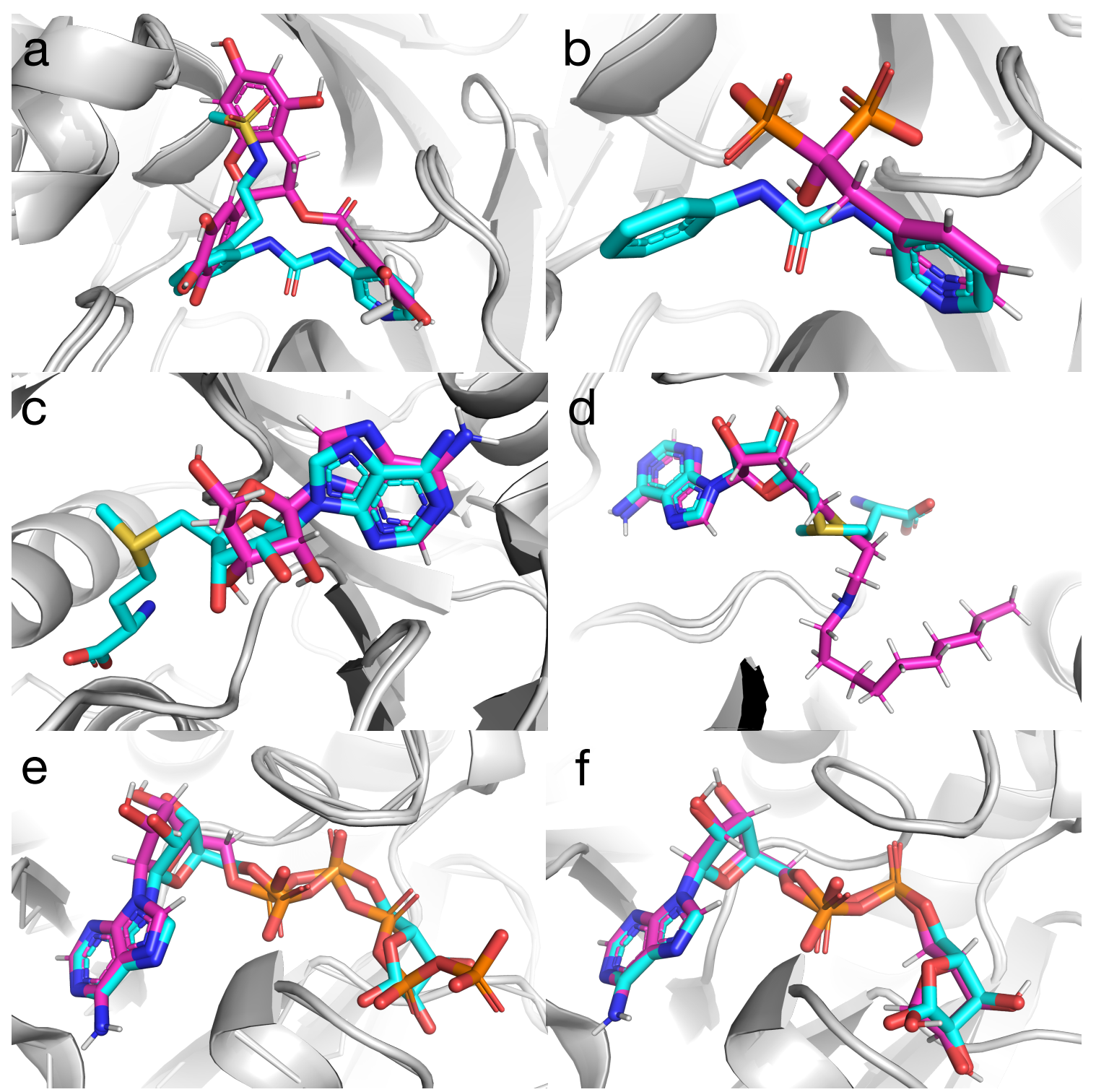

Figure 4: Structure comparison between the docked poses of hits and the fragments or ligands in the crystal structure. (purple: predicted docking poses; cyan: fragments/ligands in the crystal structures) The target in panels a to $\mathrm{c}$ is the Nsp5 3CLpro; the target in panel d is the Nsp16 2'-O-MTase. (a) Docked pose of DB12116 (Epigallocatechin gallate) compared to the bound fragment in 5R7Y and 5R83; (b) Docked pose of DB00884 (Risedronic acid) compared to the bound fragment in 5R83; (c) Docked pose of DB00640 (Adenosine) compared to the ligand in 6W4H; (d) Docked pose of DB07413 (5'-S-[2-(decylamino)ethyl]-5'-thioadenosine) compared to the ligand in 5C8T; (e) Docked pose of DB02738 (Adenosine-5'-Pentaphosphate) compared to the ligand in 6W02; (f) Docked pose of DB02059 (Adenosine-5-Diphosphoribose) compared to the ligand in 6W02. 


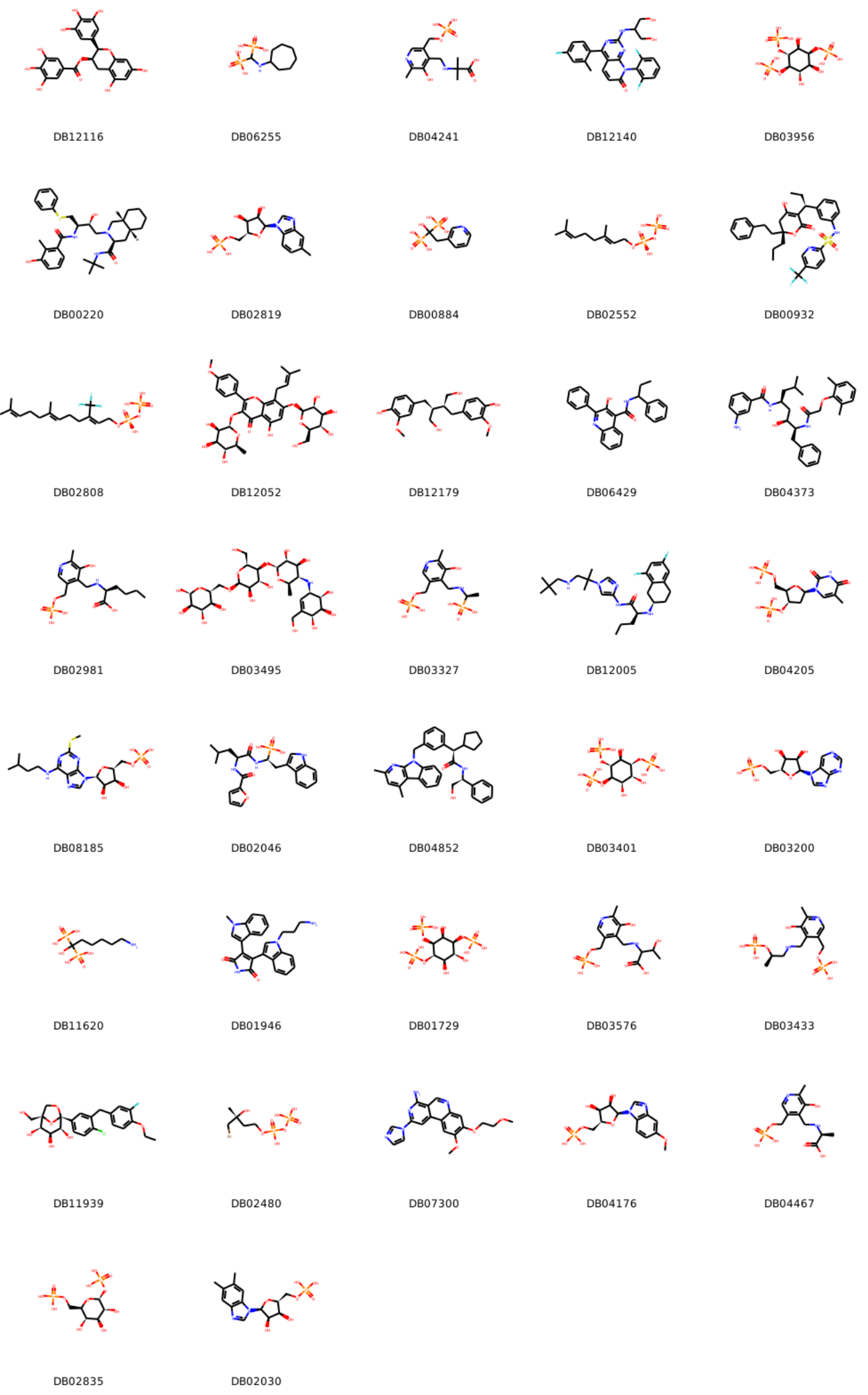

Figure 5: Manually selected designs for SARS-CoV-2 Nsp5 3CLpro. 


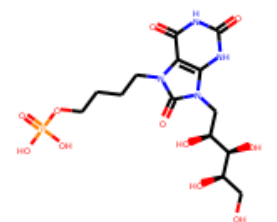

DB02711<smiles></smiles>

DB03327<smiles>C=C(C)C(C)C(C)C(C)CCCC(C)(C)C</smiles>

DB03042<smiles>Cc1cccc2c(C3NC(CCC(C)(C)C)C(C)[C@H]3C)scc12</smiles>

DB03464

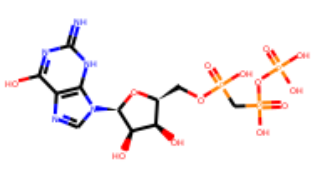

DB03532

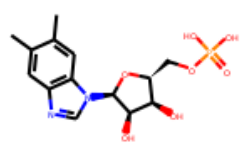

DB02030

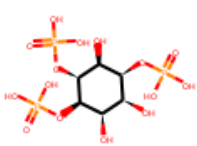

DB03401<smiles>CCC(C)C(C)C(C)CCC(C)(C)C</smiles>

DB02630<smiles>CCCC(C)C(C)CCC(C)(C)C</smiles>

DB04516<smiles>CC(CCCC(C)(C)C)C(C)C(C)C(C)CCCC(C)(C)C</smiles>

DB02992<smiles>C=C(CC)C(C)CCCC(C)(C)C</smiles>

DB01923

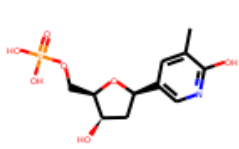

DB04060<smiles>CCC(C)CC(C)CC(C)(C)C</smiles>

DB02053<smiles>CCc1c(CCC(C)(C)C)ccc(C)c1O</smiles>

DB02209

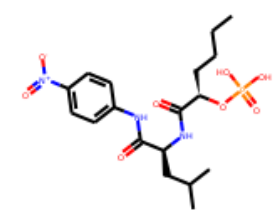

DB02276

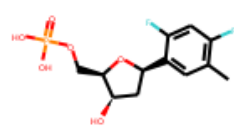

DB07652

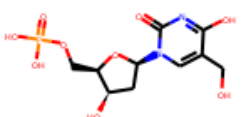

DB03846

Figure 6: Manually selected designs for SARS-CoV-2 Nsp15 Endoribonuclease 
ads

DB04703

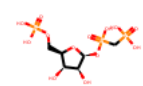

DB04294

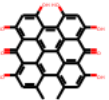

상

DB13014

DB04733

$$
\text { ard }
$$

支余.

$p^{2} d$

DB06314

DB03116

$-d^{x+4}-8$

$9^{2+2}+2$

DB03797

सㅏㅅㅏ.

DB02098

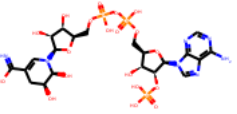

DB02319<smiles>[Te][Te][Te]</smiles>

$x$<smiles>[Te][Te][Te][Te]</smiles><smiles>[Te][Te][Te][Te]=[Te]</smiles><smiles>O[GeH2]</smiles>

DB06255<smiles>CC(C)=[Te]C(C)OC1CC1</smiles><smiles>CC(C)=[Te]OC=[Te]</smiles><smiles>[Te][Te][Te][Te]</smiles>

DB03069

DB02363

DB04097

DB01895<smiles>CCCC[Te]</smiles>

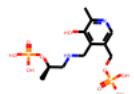<smiles>[Te][Te][Te]</smiles><smiles>OCCOCO[Te]</smiles>

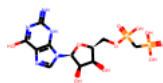

DB07873

DB03433

DB07894

DB07652

DB03486<smiles>[Y][Ca]</smiles>

DB01995

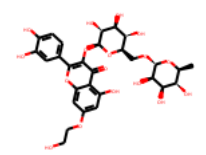

DB13764<smiles>CCCO[Te]C(=O)OCC</smiles>

DB14128

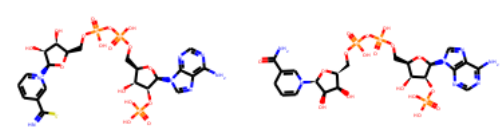

DB01763

DB02338

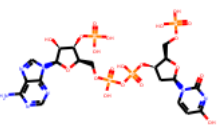

DB03186

Figure 7: Manually selected designs for SARS-CoV-2 Nsp3 ADP ribose phosphatase. 


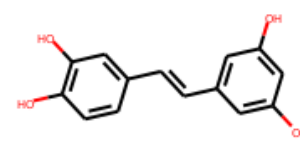

DB08399

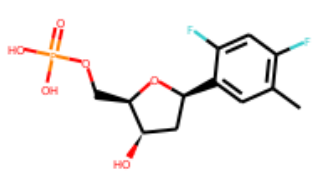

DB07652

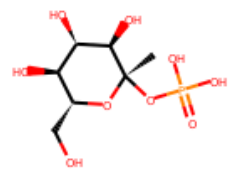

DB04195

Figure 8: Manually selected designs for SARS-CoV-2 RNA binding domain of nucleocapsid phosphoprotein

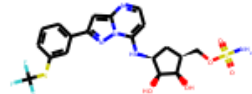

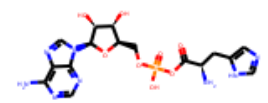

DB04201

DB15013

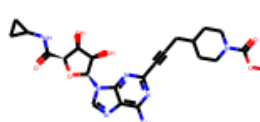

DB12295

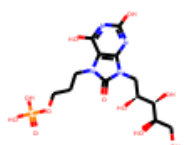

DB03022

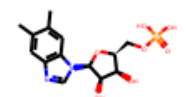

DB02030

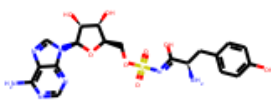

DB03325

DB14663

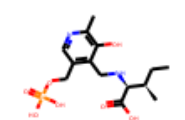

DB02635

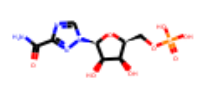

क्रा

DB02738

Figure 9: Manually selected designs for SARS-CoV-2 Nsp16 2'-O-MTase. 


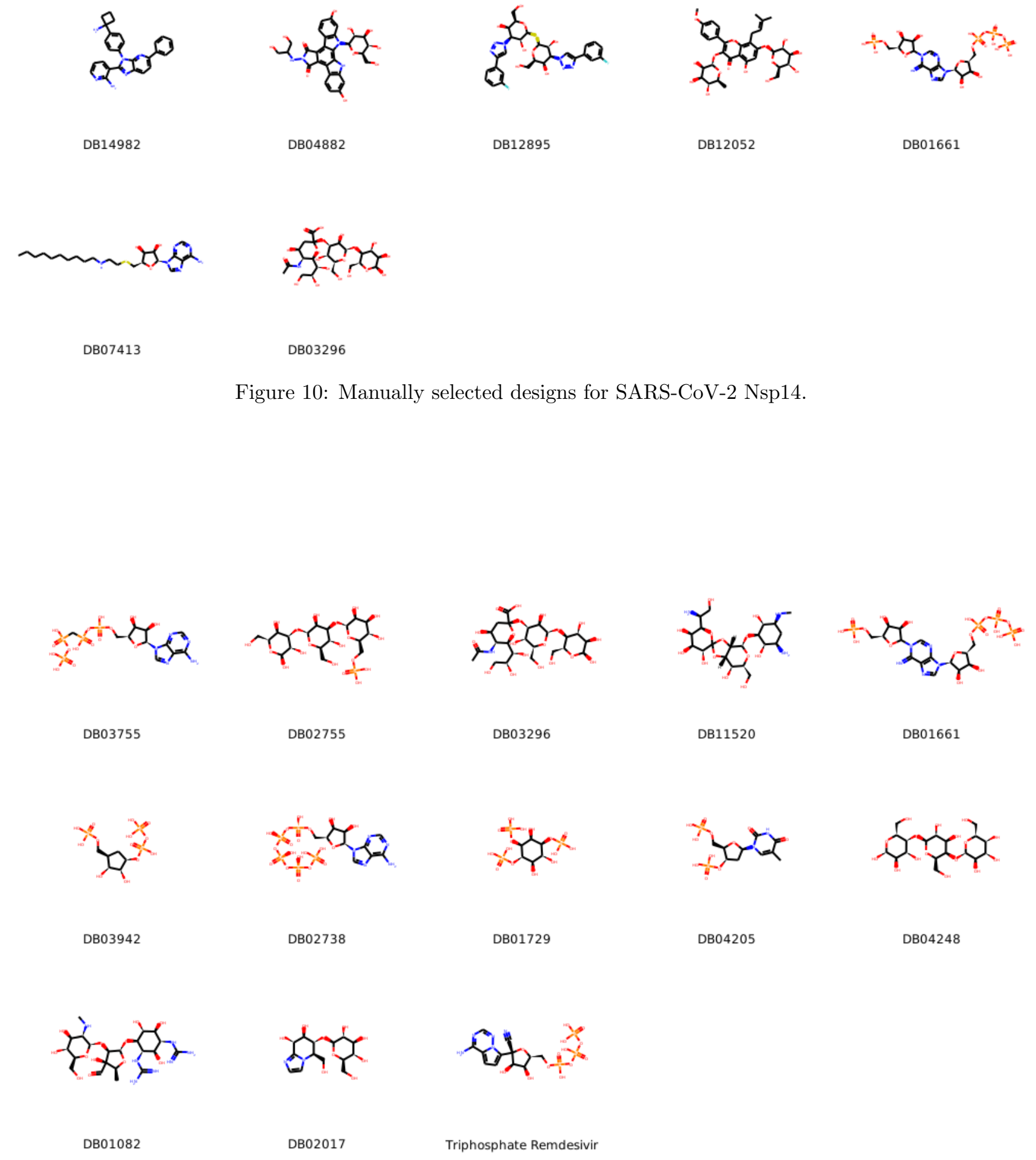

Figure 11: Manually selected designs for SARS-CoV-2 Nsp12 RdRp. 


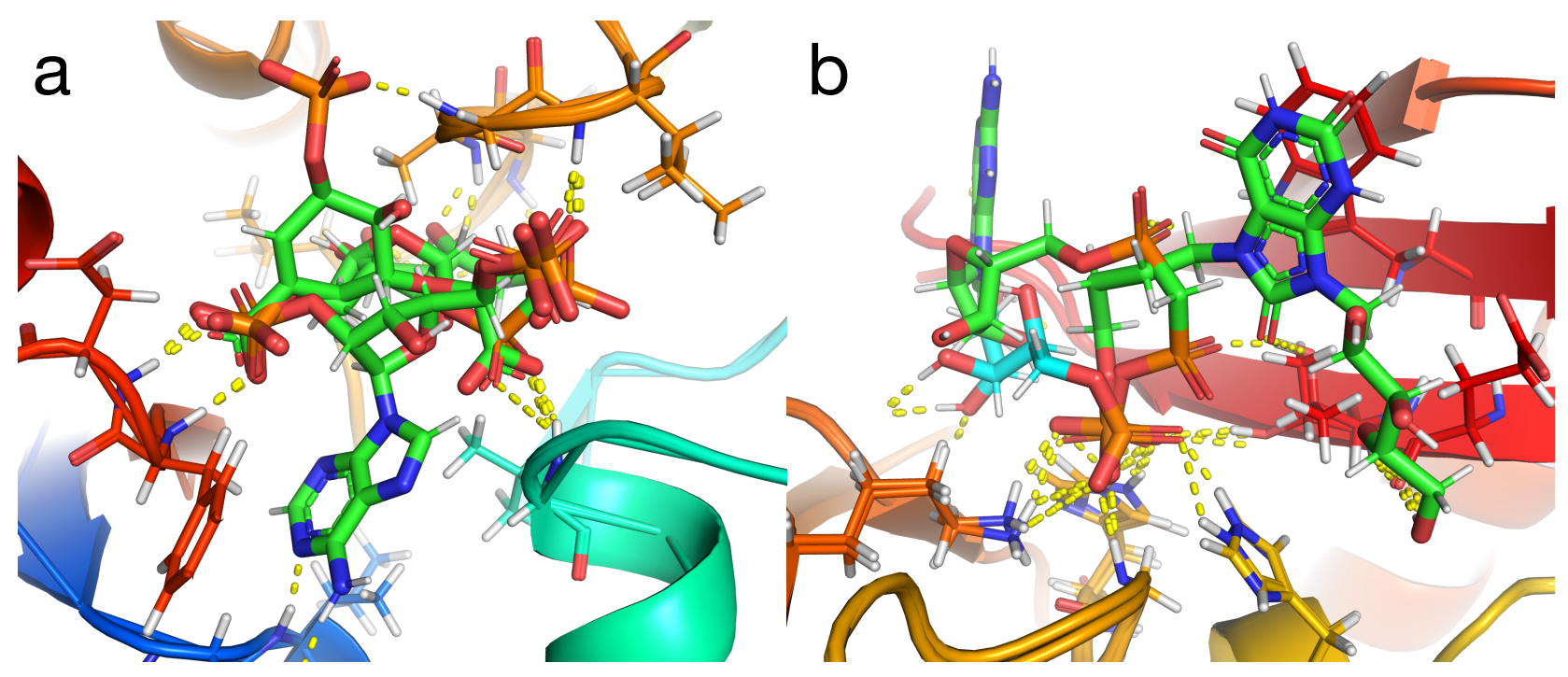

Figure 12: (a) Showing the interactions between hits DB04294, DB04733, DB03116, DB02098 and the receptor Nsp3 ADP ribose phosphatase; (b) Showing the interactions between hits DB02711, DB03532, DB04516 and the receptor Nsp15 Endoribonuclease. 